\title{
«DONNEZ-MOI TOUS LES DÉTAILS QUI CONCERNENT LE PAYS QUE VOUS HABITEZ: IL EST MIEN PAR LÀ». CARTEGGI ITALO-FRANCESI TRA IL 1789 E IL 1814
}

\begin{abstract}
EpistEur è un progetto dell'Università di Padova per il censimento e lo studio dei carteggi italo-europei nel Settecento. I dati emersi dalle prime ricerche confermano le migliori aspettative sulla fertilità e sull'ampiezza del campo d'indagine che gioverà allo studio delle relazioni internazionali, della storia diplomatica, della circolazione libraria, dello scambio culturale nonché dell'epistolografia in senso stretto, contribuendo a ridisegnare la nascita dell'idea di Europa del Settecento.

Parole chiave: epistolari, cosmopolitismo, Italia e Francia, XVIII secolo, carteggi, corrispondenza letteraria.
\end{abstract}

\section{INTRODUZIONE}

Non credo che la riflessione sui rapporti culturali tra Italia e Francia nel Settecento possa più eludere un discorso sui carteggi, sia per la loro natura di medium sia per la funzione avantestuale rispetto alla pratica della traduzione al centro dei saggi qui riuniti. La specola dell'epistolarità consente una ricerca che altrimenti non potrebbe che aspirare a precisare $o$ ampliare quadri storiografici consolidati, laddove il discorso sulle 'lettere' contribuisce a guardare al fenomeno da un punto di vista inedito rispetto ai pur ineludibili lavori di Franco Venturi 1973 (e della storiografia che ne ha accolto l'eredità; cfr. Alatri 1994) e di Norbert Jonard (1994). L' 'ipotesi di lavoro' che guida la ricerca è di ricostruire attraverso i carteggi settecenteschi italo-francesi una storia alternativa rispetto a quella che si apre nel segno della conflittualità con la polemica Orsi-Bouhours e che si chiude

\footnotetext{
*valentina.gallo.1@unipd.it
} 
con il De la littérature di Madame de Staë $1^{1}$. Ricaduta non secondaria sarà la possibilità di confrontare generi e tradizioni diverse; la lettera familiare, da parte italiana, e quella intime, da parte francese (cfr. Servais \& Ypersele 2007): cosa succede nel momento in cui le due modalità di comunicazione letteraria entrano in contatto? Una corrispondenza italo-francese è la semplice somma di due parti o non è un ente olistico, capace di generare nuovi modelli comunicativi?

«Donnez-moi tous les détails qui concernent le pays que vous habitez: il est mien par là». La scelta della citazione incipitaria è provocatoria; vorrei infatti impostare il discorso critico in termini dialettici rispetto a una vulgata storiografica che vede nella crisi dell'Illuminismo e nel periodo napoleonico la fine del cosmopolitismo settecentesco e l'affiorare dell'idea di nazione come entità non più soltanto linguistico-culturale, ma territoriale ${ }^{2}$. «Raccontatemi tutti i particolari del paese in cui siete, che, in quanto vostro, è anche mio». L'immedesimazione tra $\mathrm{i}$ due corrispondenti - la cui identità mi sia concesso di tacere ancora per poco - è tale, il legame affettivo che si mantiene vivo nella scrittura epistolare è così coinvolgente che il mittente acquista una nuova cittadinanza affettiva, superando le differenze (e le diffidenze) culturali, linguistiche, geografiche. Delabroy (1983: 377) ha rintracciato un analogo codice comunicativo che si fonda sulla «confusion à priori de la perception essentielle du mond chez [...] deux auteurs» nelle lettere intercorse tra George Sand e Gustave Flaubert, amanti e connazionali ${ }^{3}$. Tanto più sorprendente ritrovare tale simbiosi nelle lettere che intercorsero tra Madame De Staël, l'autrice del De la littérature, e Vincenzo Monti nel febbraio del 1806 (in Monti (2012: 146, lett. 110) cfr. Anne-Louise-Germaine Necker, baronessa di Staël-Holstein, a Vincenzo Monti, 9.II.1806). Ed è anche per la forza aggregante che fonda e da cui è alimentata la corrispondenza epistolare, talvolta a dispetto della diversa nazionalità del mittente $\mathrm{e}$ del destinatario, che i carteggi transnazionali possono dire qualcosa sull'ar-

${ }^{1}$ Una storia, beninteso, che si intreccia strettamente a quella dei viaggiatori e dell'immagine del nostro paese: cfr. Chevallier \& Chevallier (1983) e Hersant (1997).

${ }^{2}$ Sono categorie, quella di "nazioni culturali" e "nazioni territoriali", molto produttive per il caso italiano; cfr. per un'ampia discussione del concetto il recente lavoro di Alfonzetti \& Formica (2013); in particolare, la rassegna introduttiva (Formica 2013) e il successivo saggio problematizzante (Quondam 2013). Sebbene inerente a un arco cronologico diverso, si veda anche, per la prospettiva prescelta, Alfonzetti (2013). Una sottile analisi diacronica del concetto di 'identità nazionale' nell'ultimo decennio del Settecento è quella di Carpa (2008).

${ }^{3}$ Delabroy (1983: 379) osserva, molto acutamente, la specificità della pratica epistolare tra 'letterati': «elle n'est pas autre chose que ce désir de satisfaire, par la production d'un simulacre intime, à l'exigence de la solitude artistique sans qu'il y ait à renoncer à celle de la complicité éthique». 
ticolata e per nulla pacifica ricerca di una identità ${ }^{4}$, incrociando, dunque, il dibattito sulla debolezza congenita dell'identità italiana ${ }^{5}$.

\section{IL PROGETTO}

Il punto di partenza, dunque, sono i primi risultati di uno studio sulle relazioni epistolari tra Italia e Francia nel Settecento: il progetto, finanziato dal Dipartimento di Studi linguistici e letterari dell'Università di Padova, coinvolge, sotto il pretenzioso nome di EpistEur, studiosi di varia provenienza: Francesca Bianco, Marika Piva ed Emilio Torchio dell'Università di Padova; Corrado Viola dell'Università di Verona; Jean-Louis Haquette dell'Università di Reims ed Eric Francalanza dell'Università di Brest ${ }^{6}$.

Nella necessità di dover restringere l'arco temporale, il Settecento, a un segmento proporzionato alle dimensioni di una breve comunicazione, ho scelto di ritagliare l'arco cronologico che va dalla Rivoluzione francese al Congresso di Vienna; un periodo dalla forte articolazione interna, storica e ideologica. Devo confessare che, se fossi stata in grado di prevedere le

${ }^{4}$ Il presente discorso non può dunque che incrociare la problematica nozione di "cosmopolitismo", per la quale cfr. Formica (2013: XIV).

${ }^{5}$ Per un quadro d'insieme sulla categoria di 'italianità' e per la sua costruzione storica, cfr., oltre il già citato Carpa (2008), Cuaz (1998a), Cuaz (1998b) e Guerci (2004).

${ }^{6} \mathrm{La}$ maschera di rilevazione messa a punto con Marika Piva include una serie di campi strutturali: Mittente, Destinatario, Data cronica e topica e destinazione, lingua, area geografica, consistenza, fonte bibliografica (e natura dell'intervento: edizione, studio o catalogo) e/o localizzazione del manoscritto, presenza di inclusi (poesie, lettere, documenti, ecc.) e un unico campo tematico, in cui far confluire generiche parole chiave (guerra, politica, letteratura, traduzioni, rapporti personali, ecc.). Va infatti precisato che l'oggetto della catalogazione, allo stato attuale, non è la singola lettera, ma il carteggio: l'ampiezza di alcuni di questi (penso, ad esempio, a quello Manzoni-Fauriel) scoraggia, infatti, una schedatura a livello di singola epistola. Un diverso approccio alla catalogazione epistolare è quello applicato da Grassi (1983) a 260 lettere. Grassi parte da quattro variabili fondamentali che descrivono il profilo dell'individuo (l'origine geografica, l'origine sociale, il tipo di relazione (generi sessuali) e il tipo di relazione affettiva); la tipologia epistolare; il livello linguistico (grafia, ortografia, provincialismo, stile) e i temi (sé, l'altro, le notizie sociali, la letteratura, la coppia, l'istituzione, la religione); di ogni tema si registra la presenza o l'assenza, il modo in cui viene trattato (oggettivo: menzione di un'opera; soggettivo: attitudine dell'autore positiva o negativa). Cfr. Grassi (1994). In questo secondo lavoro, l'autrice raccoglie i dati di una schedatura contenutistica di 1100 lettere 'private' francesi scritte tra il 1700 e il 1850, in base alle categorie di: 'notizie sociali e familiari', 'occupazioni personali', notizie politiche, notizie relative all' educazione dei figli, allusioni alla religione, confidenze personali, notizie relative al matrimonio e alla vita di coppia, informazioni artistiche, letterarie ed erudite. 
difficoltà che tale découpage avrebbe comportato, avrei operato diversamente, per molteplici ragioni: a cominciare dalla periodizzazione interna al 1789-1814, necessariamente a posteriori degli eventi e da un'ottica politico-unitaria che non è certo quella dei corrispondenti di cui ci occupiamo; i quali, oltretutto, dislocati in realtà storico-politiche profondamente diverse, vivono quel quarto di secolo da prospettive ideologiche differenti e con vistosi sfasamenti temporali ${ }^{7}$.

A questo ordine di problemi si sommano l'impasse materiale (le deficienze del sistema dei corrieri d'antico regime, rese ancor più drammatiche dalle tensioni politiche in corso e dal venir meno di un'autorità centrale capace di garantire la 'normalità' della trasmissione epistolare) e, di contro, l'inasprirsi del controllo postale e il moltiplicarsi dei censori ${ }^{8}$. Lascio la parola a Monti che informa, smarrito, Madame de Staël (è il giugno del 1805):

Vi ho esposto lo stato infelice dell'animo mio nella seconda lettera che di qui vi ho scritta e diretta a Coppet secondo il modo da voi indicatomi. Voglia il cielo che questa ingenua confessione dei miei sentimenti si sia salvata. Il corriere che la portava (ed era un corriere di Talleyrand) è stato assassinato tra Lodi e Milano, e i pieghi che portava tutti dispersi e gettati in mezzo alla strada. La presente l'indirizzo a Fortis secondo la vostra istruzione, e terrò questa strada nell'avvenire (Monti 1928: 424, lett. 926, 25.VI.1805).

Sebbene non sempre la comunicazione epistolare andasse incontro a esiti così drammatici, dobbiamo presupporla sempre triangolare: la lettera doveva essere scritta in modo da poter passare al vaglio di un indiscreto e rigido censore. Così, ad esempio, Giambattista Bodoni si rivolge nel 1794 ad Antoine-Augustine Renouard, bibliofilo e mercante di libri francese:

A parlar franco debbo dirvi che io temevo assai che elleno [le lettere] non giungessero al lor destino; molte dicerie si vanno dagli ignoranti o da' malevoli divulgando sulla incertezza delle poste, sulla apertura delle lettere, sulla intermissione del commercio, che io ho fissato di nulla più credere, e proseguir a rispondervi con quella candida apertura di cuore che è in me ingenita, e di null'altro m'immischierò mai che di affari letterari e di libri e di edizioni. In questa guisa contenendomi, son certissimo che, quand'anche le mie lettere venissero ad essere aperte e lette colla più esatta scrupolosità, non anderanno mai smarrite, tanto più che io sono persuaso che le

${ }^{7}$ Cfr. le pagine di Ricuperati (2003: 34-36); sull'‘anomalia' siciliana, cfr. Renda (1996); sulla situazione degli stati preunitari di fronte al problema di un'identità nazionale, cfr. il volume miscellaneo De Benedictis et al. (2012).

${ }^{8}$ Cfr. i volumi dell'Istituto di studi storici postali, in particolare: Fedele (1996) e Caizzi (1993), per quanto interessati all'insorgere di una regolamentazione del servizio piuttosto che a rilevare le falle del sistema. 
persone destinate a presiedere agli uffizi delle poste sono soggetti di conosciuta probità ed onoratezza, né ometteranno mai di dar corso a que' fogli che tendono all'incremento delle lettere, delle arti e ad un vicendevole reciproco commercio (in Boselli (1927: 37): Bodoni a Renouard, 18.III.1794).

\section{Cui fa eco Renouard:}

Oui, sans doute, notre correspondance littéraire n'éprouvera aucun obstacle de la part des Français. Amis des arts, passionnés pour tout ce qui est beau, aimant la vertu malgré le système d'immoralité que de vils conspirateurs avaient essayé, mais en vain, de propager dans notre chère patrie, jamais les Français ne chercheront à inquiéter, à tourmenter ceux qui, contents de faire passer en France leurs idées littéraires, respecteront les lois et le gouvernement que la volonté d'un peuple puissant et libre y a établis (in Boselli (1927: 39): Renouard a Bodoni, 15.IV.1794).

Ma la retorica della blandizie non dovette essere sufficiente, se allo scadere dello stesso anno, Bodoni doveva confessare:

Io vi ho scritto diverse lettere ne' mesi passati, ma, non avendone mai avuto riscontro, torno adesso a ripetervi brevemente quanto vi avea significato nelle dette mie. E se mai anche la presente avesse la sfortuna di andare smarrita, non saprei più qual mezzo tenere per farvi giungere in avvenire le mie lettere: mi resta solo qui di pregare chiunque potesse aprire e leggere questa mia di mandarla poscia al suo destino, giacché non tratta di altro il presente foglio che di faccende letterarie e che hanno rapporto al solo commercio librario (in Boselli (1927: 43): Bodoni a Renouard, 29.XII.1794).

In questo contesto la comunicazione, e soprattutto quella scritta e transnazionale, si fa cifrata, reticente, quando non addirittura antifrastica, come dimostra bene la lettera di Bodoni: interpretarne il senso diventa operazione delicata, necessaria di una precisa contestualizzazione, della ricostruzione, cioè, delle finalità epistolari, delle identità dei corrispondenti e di quel "Convitato di pietra" che è lo scomodo e onnipresente ufficiale postale 9 .

L'argomento definitivo che avrebbe dovuto dissuadermi dall'eleggere come 'campo di sperimentazione' l'arco cronologico a cavaliere tra il Sette e l'Ottocento, è la damnatio memoriae che colpì, alla caduta di Napoleone, una stagione breve e concitata della nostra tradizione, e che di certo non giovò alla custodia dei documenti, generosi testimoni di rapporti epistolari politici o amicali che ai nuovi governanti potevano apparire delittuosi.

${ }^{9}$ E sono prudenze ermeneutiche che raccomandava alcuni anni fa anche Romagnani (2000), e sulle quali è tornato più di recente (Romagnani 2011: 12-13). 


\section{CARTEGGI EUROPEI E STORIOGRAFIA}

Discontinuità storica del periodo, natura cifrata della comunicazione epistolare, deficienze e permeabilità del sistema postale, dispersione documentaria hanno dunque ostacolato da parte della storiografia, anche quella attenta alla storia delle idee, l'opportuna valorizzazione dei carteggi transnazionali: il pioneristico lavoro di Paul Hazard (1995), per esempio, passa in rassegna le gazzette, i giornali, le traduzioni; una considerevole mole di materiale che non include, tuttavia, le corrispondenze 'familiari' (cfr. anche Galasso 1989). E se è vero che Hazard scriveva all'inizio dello scorso secolo, quando gli epistolari giacevano in buona parte sepolti negli archivi familiari o nelle biblioteche, l'intelligente contributo di Jonard (1994) o del compianto Guerci (2008) ignorano le relazioni epistolari o si limitano a sfruttare - Guerci - i dispacci diplomatici degli ambasciatori veneziani a Parigi ${ }^{10}$.

Alla base di questa pigrizia storiografica ci sono precise ragioni ideologiche: dapprima una storiografia nazionale restia a riconoscere che l'identità italiana si fosse costruita anche attraverso il confronto con le altre, più forti e mature, realtà politiche europee (Guerci 2004). Poi, quando quell'Europa politica è stata costruita, e nonostante i tentativi di un Umberto Eco o di un Asor Rosa, ci si è fermati ai grandi affreschi, a dinamiche sistemazioni, ben al di qua, tuttavia, di una ricerca d'archivio o di prima mano. E ciò nonostante è indubbio il valore innovativo dei lavori che, proprio grazie a una lettura attenta dei carteggi, hanno consentito di superare la categoria storiografica dell'influence e di accogliere quella dell'échange, giustamente al centro dei lavori di Waquet (1989). Sarà per questo che i carteggi transnazionali editi si contano, per il periodo preso in esame, sulle dita di una sola mano: Manzoni-Fauriel (Manzoni 2000), Bettinelli-Féraud (Finoli 1965), e poco altro: lo stesso carteggio tra la de Staël e Monti non è stato ricomposto bibliologicamente, ma va idealmente assemblato attraverso la lettura dei due, parziali, epistolari a stampa (cfr. Necker 1985, Monti 1928, Monti 2012, Lanfredini 1946, Lanfredini 1947). Ed è tanto più grave questa lacuna, in quanto proprio in questo arco temporale l'idea di patria si fa progetto politico (cfr. Cuaz 1998b: 86-87).

\section{I FLUSSI CULTURALI INTERNAZIONALI}

Sarà necessario, tuttavia, tenere conto anche di dati quantitativi: i carteggi a stampa sull'asse italo-francese nel periodo preso in esame sono

${ }^{10}$ Per alcune considerazioni sulla corrispondenza diplomatica, cfr. De Raymond (1983); per un saggio applicativo, invece, cfr. Scarabello (1992). 
pochi; il flusso culturale, infatti, già da qualche decennio si è orientato verso Nord-Est, verso l'impero e il mondo tedesco, anche conseguentemente al nuovo equilibrio politico e al maggior peso che Vienna acquista nella politica italiana. A partire, ma sono prime ipotesi che andranno confortate dai dati che emergeranno dall'analisi dell'intero secolo, dagli anni Sessanta, venuta meno la spinta propulsiva dell'Illuminismo francese, il grosso della corrispondenza postale non valica più il Moncenisio o il Monginevro, ma batte la strada del Brennero. È sufficiente sfogliare l'epistolario di Cesare Beccaria (1738-1794) - in cui spiccano la quantità e soprattutto la qualità dei corrispondenti francesi - e confrontarlo con l'indice dei corrispondenti di Cesarotti (più giovane sì di otto anni, ma ben più longevo: morirà, infatti, nel 1808), di Alfieri (nato nel 1749 e morto 1803), di Foscolo ${ }^{11}$, di Monti, ecc. In tutti si registrerà un forte restringimento dell'orizzonte internazionale, parzialmente bilanciato da sporadiche presenze austro-tedesche (anche grazie, soprattutto a livello scientifico, alla forza attrattiva rappresentata dall'Accademia di Berlino).

\section{GLI EPISTOLOGRAFI}

\subsection{I mercanti della cultura}

Ma chi, o meglio, quali gruppi sociali, tra il 1789 e il 1814, intrattennero rapporti epistolari con la Francia? In primo luogo i mercanti, e i mercanti di libri, nel settore che più ci interessa. È ben nota la ricchezza della Correspondance della Societé Tipographique di Neuchâtel, studiata da Robert Darnton $(1979,1986)$ : una miniera di informazioni sul commercio e sulla circolazione della cultura nell'Europa d'antico regime. Per gli anni che ci riguardano, e salve le necessarie proporzioni, soccorre, ad esempio, il già ricordato carteggio Bodoni-Renouard, attraverso il quale è possibile saggiare la ricezione della letteratura italiana in Francia e viceversa. Mediatore tra i due era stato il libraio francese di stanza a Parma Jacques Banchon, che aveva fornito a Renouard il catalogo bodoniano. Il carteggio, edito da Antonio Boselli tra il 1927 e il 1930, conta 73 missive (1791-1812), tra originali e minute, conservate nel fondo Bodoni della Biblioteca Palatina di Parma.

Lo scambio epistolare si apre con una lettera di Renouard a Bodoni, in cui il bibliofilo francese si presenta come esponente di un pubblico d'élite, che ammira la pulizia e l'eleganza formale dell'officina bodoniana, ma che accusa nel catalogo dello stampatore italiano un eccessivo numero di baga-

${ }^{11} \mathrm{Nel}$ ricco epistolario foscoliano si conoscono solo due lettere a un francese, Guinguené, sul quale, cfr. Zini (1930). 
telle, le famigerate raccolte poetiche encomiastiche; dall'officina bodoniana, precisa Renouard nella missiva seguente, i suoi 'amici' vorrebbero un beau Tasso, un Petrarca, un Ariosto quello del Furioso ${ }^{12}$, e aggiunge: «quelq'un de beaucoup de goût me recommande de vous engager à imprimer comme les Odi di Parini trois poemetti du même intitulés Il Mattino, il Mezzo-giorno e La Sera. Je ne les ai pas lus, mais on m'assure qu'il sont très curieux et valent la peine de faire un petit volume qu'on annexerait aux Odes» (in Boselli (1926: 246): Renouard a Bodoni, lett. 2, 6.V.1792; per il séguito del carteggio vedi Boselli 1927, 1930). Bodoni, per parta sua, si dice sollecito, nonostante $\mathrm{i}$ «tempi calamitosi», a eseguire le commissioni di Renouard, e interessato a stampare anche qualche classico francese di cui il corrispondente avrà la compiacenza di inviargli l'esemplare per la stampa. Renouard è generoso di consigli: nel 1794 invia un elenco di autori quanto mai eloquente sulla corrispondenza tra un determinato gruppo sociale - realista anche in piena rivoluzione - e il canone letterario corrispondente: Boileau (nelle edizioni Didot l'ainé), Racine, La Fontaine, e soprattutto Bossuet, i cui Discours sur l'histoire universelle saranno un valido aiuto per leggere la storia contemporanea. Da Bodoni Renouard acquista la lettera critica di Esteban Arteaga su Orazio, le poesie di Clemente Bondi, la Liberata e l'Aminta, ma anche le Stagioni di Thomson, Dante, gli scritti di Serassi Sulla controversia del Tasso e dell'Ariosto, il Saggio di Poesie di Morelli, le Anacreontiche di Savioli, gli Scherzi poetici e pittorici di G. G. De Rossi, il Pastor fido, Poliziano, l'opera di Carlo Luigi Benvenuto Robbio conte di San Raffaele e un classico dell'antilluminismo: le Disgrazie di Donna Urania. Le circostanze storico-politiche rappresenteranno un ostacolo non indifferente al commercio transalpino, che tuttavia si interromperà, dopo la visita di Renouard all'officina bodoniana (1809), solo poco prima della morte di Bodoni.

\subsection{L'internazionale religiosa}

Un altro vivace e assai interessante gruppo sociale coinvolto in corrispondenze transalpine è rappresentato dall' 'internazionale' religiosa, che negli ultimi trent'anni del secolo, in occasione dello scontro tra giansenisti ed ex-gesuiti, aveva spinto traduttori e giornalisti a un serrato confronto tra la realtà italiana e quella francese. In questi ambienti la Rivoluzione francese era stata letta come la diretta conseguenza della propaganda massonica, di quella dei philosophes e dei giansenisti, rafforzando i legami ideali tra

${ }^{12}$ Sulle edizioni bodoniane, cfr. Brooks (1927) e Giani (1948); un pregevole lavoro di edizione dei carteggi tra Bodoni e i corrispondenti spagnoli è quello avviato da Pedro M. Cátedra, consultabile sul portale bibliotecabodoni.net. 
i papisti di qua e di là delle Alpi. L'approvazione da parte dell'Assemblea nazionale, nel luglio del 1790, della costituzione civile del clero e, nel novembre dello stesso anno, l'imposizione del giuramento di fedeltà alla medesima, costrinse i religiosi francesi refrattari a cercare rifugio soprattutto, ma non solo, a Roma, come insegnanti di lingua francese e traduttori. Al centro della rete settentrionale dei réfugiés c'è, anche per ragioni geografiche, il mantovano Saverio Bettinelli, il cui epistolario, come ha documentato Agosta del Forte (1970), annovera svariati corrispondenti francesi.

Mole, continuità e rilievo fanno del carteggio con Féraud, ad esempio, uno scambio denso e proficuo, il cui valore storiografico ha consentito a Finoli $(1965: 241 ; 1968)$ di mettere in discussione e ripensare il cliché storiografico, formulato da Paul Hazard, degli émigrés francesi durante la rivoluzione come del tutto assorbiti dalla propria condizione di esuli e dalle strategie per rientrare in Francia (cfr. Brun 1951; e contra Hazard 1995: 18). Féraud, linguista e compilatore del Dictionaire critique de la langue française, fu un profondo conoscitore della lingua italiana; nel carteggio che intrattenne con Bettinelli sottopose al mantovano le traduzioni della Secchia rapita di Tassoni e della lettera di Bettinelli su Algarotti; mentre l'italiano ricambiava inviandogli, e chiedendogliene lettura, le sue Lettere sugli epigrammi. Lo scambio fu a tal punto intenso da suggerire a Féraud un progetto editoriale che, benché mai realizzato, testimonia la fertilità di una pur breve stagione (Féraud rientrò in Francia alla fine del 1798):

\footnotetext{
Si nous retournons l'anée prochaine en France et que l'amour des Lèttres y renaisse, je compte ouvrir une souscription de Mélanges de Littérature Italiènne, en vers et en prose, traduits en français. J'y embrasserai à peu prês tous les genres. Je donerai deus petits volumes de six ou huit feuilles chaque mois, dont l'un comprendra la litérature profane et l'autre la sacrée. On sera le maitre de les prendre tous les deus ou de n'en prendre qu'un. J'ai quelques avances en poésie; pour la prose je me métrai facilement au courant. Si la chose réussit, je vous consulterai sur le chois des livres à traduire; mais d'avance je pense que vous seuls vous me fournirez de la matière pour plus d'un année (in Finoli (1965: 260): Jean-François Féraud a Saverio Bettinelli, 26.XII.1794).
}

\subsection{Armi e lettere}

L'ultimo gruppo sociale che instaura rapporti epistolari significativi è quello composto dai militari francesi che attraversarono il nostro paese a capo degli eserciti napoleonici. Appartenenti per lo più ai ranghi dell'aristocrazia, con alle spalle una cultura che, se non comportava la conoscenza della lingua, includeva però almeno un'infarinatura della letteratura classica, svolsero, politicamente, un ruolo chiave nel tentativo di conquistare 
gli animi e le simpatie dei salotti e delle accademie italiane (in Catalani (2016: 208), lett. Bettinelli 70, vedi Saverio Bettinelli a Giovanni De Lazara, 10.VI.1801). Il loro più celebre rappresentante è probabilmente il generale Sextius-Alexandre-François Miollis (1759-1828), a comando della piazza mantovana, protettore delle arti e delle lettere, promotore delle celebrazioni virgiliane a Mantova e di quelle ariostesche a Ferrara. L'epistolario di Miollis, regestato nel $1961 \mathrm{da} \mathrm{H}$. Aureas, è disperso tra gli archivi centrali francesi e quelli periferici italiani: tra i suoi corrispondenti si contano, tra gli altri, Cesarotti e Bettinelli, al quale inviò 98 lettere tra il 1797 e il 1808. Il caso di Miollis è emblematico, credo, di quanto lavoro resti ancora da fare se si vuole tentare di ricostruire una stagione culturale in cui l'evento bellico non fu soltanto rapina e conquista, ma anche occasione per la costruzione di un dialogo che le future generazioni avrebbero preferito dimenticare.

\section{LE QUESTIONI}

A valle di questo detour epistolare tardo-settecentesco, vorrei riflettere brevemente su alcuni aspetti della comunicazione per lettera e sul rapporto carteggi-storia. Sarebbe un errore, infatti, investire le carte in oggetto del compito di ribaltare le sistemazioni storiografiche consolidate. Altrettanto grave, tuttavia, sarebbe sottostimarle, perché la storiografia che non compulsa i carteggi non solo non coglie in atto un aspetto della circolazione internazionale delle idee ${ }^{13}$ - per la quale sono stati proposti dei modelli reticolari (cfr. Beaurepaire 2006, Beaurepaire et al. 2006) - ma non è in grado di rilevare il contributo che la scrittura per lettera apporta all'epocale riconoscimento di uno statuto estetico del sé (cfr. Grassi 1994), ignora della storia delle idee la loro germinazione nella 'coltura' trans-nazionale ${ }^{14}$. Non si tratta neanche di rivendicare la ricchezza di una storiografia dell'affettività; guardare a quegli anni dalla specola dei carteggi, di quelli minori, come da quella dei grandi, consente infatti di osservare in atto un duplice processo, che si costruisce dal basso, dal contatto personale, dalla rete di conoscenze comuni: da una parte, e questo vale soprattutto per la realtà italiana, la messa a fuoco della propria identità nel dialogo con un'altra più forte e storicizzata; nelle lettere tra Monti e Madame de Stäel si incontrano e si confrontano due culture, e un forte pregiudizio che il viaggio in Italia

${ }^{13} \mathrm{Si}$ vedano i risultati di questa apertura in Bergamasco \& Bossis (2007); e in un lavoro prezioso come quello di Chapron (2008).

${ }^{14}$ Una specifica valorizzazione dei carteggi è promossa in Italia dal C.R.E.S. di Verona e in Francia dal Centre Culturel International de Cerisy la Salle, di cui si vedano gli atti dei convegni del 1983-1994: Bonnat et al. (1986), ma cfr. anche Bossis (1994), Bossis \& Porter (1990), Bray \& Strosetzki (1995), Chartier (1991) e Greimas (1988). 
e la corrispondenza con Monti contribuiranno a fiaccare. Dall'altro lato matura una cultura del confronto e del dialogo che toccherà le vette nel carteggio tra Manzoni e Fauriel, con l'accenno al quale vorrei concludere questa breve rassegna: cosa sarebbe stato Manzoni senza il dialogo con Fauriel? Insomma, permettetemi di immaginare con sgomento un Ottocento italiano in cui fosse mancato a Manzoni la possibilità di bruciare, proprio nel carteggio con Fauriel, l'aspirazione a un teatro tragico.

\section{BIBLIOGRAFIA}

Agosta del Forte, E. (1970). Corrispondenti francesi di Saverio Bettinelli. Mantova: Ponte Vecchio.

Alatri, P. (1994). Comprensioni e incomprensioni tra italiani e francesi nel periodo illuminista. In C. Bertelli (a cura di), I francesi e l'Italia (pp. 25-32). Milano: Scheiwiller.

Alfonzetti, B. (2013). Dell'Italia o la doppia nazione. Prospettive politicoletterarie negli anni 1700-1748. In B. Alfonzetti \& M. Formica (a cura di), L'idea di nazione nel Settecento (pp. 31-49). Roma: Edizioni di Storia e Letteratura.

Alfonzetti, B. \& Formica, M. (a cura di). (2013). L'idea di nazione nel Settecento. Roma: Edizioni di Storia e Letteratura.

Beaurepaire, P.-Y. (2006). La plume et la toile. Pouvoirs et réseaux de correspondance dans l'Europe des Lumières. Saint-Étienne: Publications de l'Université de Saint-Étienne.

Beaurepaire, P.-Y. et al. (2006). Réseaux de correspondance à l'âge classique (XVI $-X V I I I^{e}$ siècle). Saint-Étienne: Publications de l'Université de Saint-Étienne.

Bergamasco, L. \& Bossis, M. (a cura di). (2007). Archive épistolaire et histoire. Cerisy: Connaissances et Savoirs.

Bonnat, J.-L. et al. (a cura di). (1986). Des mots et des images pour correspondre, Actes du II colloque international "Les correspondances", Nantes 13-15 septembre 1984. Nantes: Université de Nantes.

Boselli, A. (1926). Corrispondenza di Antonio-Agostino Renouard con Giambattista Bodoni. La bibliofilia, 28, 241-258.

Boselli, A. (1927). Corrispondenza di Antonio-Agostino Renouard con Giambattista Bodoni. La bibliofilia, 29, 37-46, 93-108 e 313-331.

Boselli, A. (1930). Corrispondenza di Antonio-Agostino Renouard con Giambattista Bodoni. La bibliofilia, 32, 222-231, 365-373 e 450-457.

Bossis, M. (a cura di). (1994). La lettre à la croisée de l'individuel et du sociale, Actes du colloque Paris, Institut International de la Recherche, décembre 1992. Paris: Kimé. 
Bossis, M. \& Porter, C. A. (a cura di). (1990). L'épistolarité à travers les siècles. Geste de communication et/ou d'écriture, Actes du colloque international Centre Culturel International de Cerisy 1-18 luglio 1987. Stuttgart: Steiner.

Bray, B. \& Strosetzki, C. (a cura di). (1995). Art de la lettre, art de la conversation à l'époque classique en France, Actes du Colloque de Wolfenbuttel, octobre 1991. Paris: Klincksieck.

Brooks, H. C. (1927). Compendiosa bibliografia ... di edizioni bodoniane. Firenze: Barbera.

Brun, A. (1951). L'Abbé Féraud et l'Italie. Revue de littérature comparée, $25,338-343$.

Caizzi, B. (1993). Dalla posta dei re alla posta di tutti. Territorio e comunicazioni in Italia dal XVI secolo all'Unità. Prato: Istituto di studi storici postali.

Carpa, C. (2008). Questione nazionale e identità italiana nel periodo rivoluzionario (1789-1802). In D. Baneli et al. (a cura di), Dall'origine dei Lumi alla rivoluzione. Scritti in onore di Luciano Guerci e Giuseppe Recuperati (pp. 125-143). Roma: Edizioni di Storia e Letteratura.

Catalani, G. (2016). Tra Mantova e Padova. Arte e storia nel carteggio tra Saverio Bettinelli e Giovanni De Lazara (1795-1808), con un saggio introduttivo di L. Caburlotto e una nota di S. Ghini. Verona: QuiEdit.

Chapron, E. (2008). L'Europe à Nîmes: les carnets de Jean-François Séguier (1732-1783). Nîmes: A. Barthélemy.

Chartier, R. (a cura di). (1991). La correspondance. Les usages de la lettre au XIX $X^{e}$ siècle. Paris: Fayard.

Chevallier, E. \& Chevallier, R. (1983). Iter Italicum. Les voyageurs français à la découverte de l'Italie ancienne. Ginevra: Slatkine.

Cuaz, M. (1998a). L'identità ambigua. L'idea di nazione tra storiografia e politica. Rivista storica italiana, 110, 2, 573-641.

Cuaz, M. (1998b). L'immagine dell'Italia nella cultura europea del Settecento. Geographia antiqua, 7, 67-88.

Darnton, R. (1979). The Business of Enlightenment. A Publishing History of the Encyclopédie 1775-1800. Cambridge Mass.: Belknap of Harvard University Press (trad. it. Darnot, R. (1999). Il grande affare dei Lumi. Storia editoriale dell 'Encyclopédie 1775-1800. Milano: S. Bonnard).

Darnton, R. (1986). Le marché littéraire français vu de Neuchâtel. In J. Rychner \& M. Schulp (a cura di), Aspects du livre neuchâtelois (pp. 59-75). Neuchâtel: BPU.

De Benedictis, A. et al. (a cura di). (2012). Nazioni d'Italia. Identità politiche e appartenenze regionali fra Settecento e Ottocento. Roma: Viella. 
De Raymond, J.-F. (1983). Correspondance et correspondances diplomatiques. In M. Bossis e J.-L. Bonnat (a cura di), Les correspondances: problématique et économie d'un genre littéraire: écrir, publier, lire, Actes du Colloque international, Nantes 4-7 ottobre 1982 (pp. 126142). Nantes: Publications de l'Université de Nantes.

Delabroy, J. (1983). Le courant de la plume. Mythe et vérité de la correspondance (l'exemple Gustave Flaubert - George Sand). In M. Bossis e J.-L. Bonnat (a cura di), Les correspondances: problématique et économie d'un genre littéraire: écrir, publier, lire, Actes du Colloque international, Nantes 4-7 ottobre 1982 (pp. 375-391). Nantes: Publications de l'Université de Nantes.

Fedele, C. (1996). La voce della posta. Comunicazione e società nell'Italia napoleonica. Prato: Istituto di studi storici postali.

Finoli, A. M. (1965). Un corrispondente francese del Bettinelli «italianisant» e filologo. In Studi di letteratura, storia e filosofia in onore di Bruno Revel (pp. 235-264). Firenze: Olschki.

Finoli, A. M. (1968). Inediti francesi del Bettinelli. Lettere italiane, 20, $391-402$.

Formica, M. (2013). Patria e nazione nel Settecento. In B. Alfonzetti e M. Formica (a cura di), L'idea di nazione nel Settecento (pp. VII-XXII). Roma: Edizioni di Storia e Letteratura.

Galasso, G. (1989). Rivoluzione francese e lettere italiane: la prima grande opera di Paul Hazard. Rivista storica italiana, 110, 293-302.

Giani, G. (1948). Catalogo delle autentiche edizioni bodoniane. Milano: Conchiglia.

Grassi, M.-C. (1983). Un exemple d'analyse sérielle: les correspondances intimes de la noblesse française. In M. Bossis e J.-L. Bonnat (a cura di), Les correspondances: problématique et économie d'un genre littéraire: écrir, publier, lire, Actes du Colloque international, Nantes 4-7 ottobre 1982 (pp. 239-254). Nantes: Publications de l'Université de Nantes.

Grassi, M.-C. (1994). L'art de la lettre au temps de la «Nouvelle Héloïse» et du Romantisme. Genève: Slatkine.

Greimas, A.-J. (a cura di). (1988). La lettre: approches sémiotique, Actes $d u V^{e}$ Colloque interdisciplinaire en collaboration avec l'Association Suisse de Sémiotique. Fribourg-Suisse: Éditions Universitaires.

Guerci, L. (2004). Il triennio 1796-1799 e la «Repubblica itala». In U. Levra (a cura di), Nazioni, nazionalità, stati nazionali nell'Ottocento europeo, Atti del LXI Congresso di storia del Risorgimento italiano Torino, 9-13 ottobre 2002 (pp. 59-103). Torino: Comitato di Torino dell'Istituto per la Storia del Risorgimento italiano/Carocci. 
Guerci, L. (2008). Uno spettacolo non mai più veduto nel mondo. La Rivoluzione francese come unicità e rovesciamento negli scrittori controrivoluzionari italiani (1789-1799). Torino: UTET.

Hazard, P. (1995). Rivoluzione francese e lettere italiane (1789-1815), a cura di P. A. Borgheggiani. Roma: Bulzoni (ed. orig. 1910).

Hersant, Y. (1997). Italies. Anthologie des voyageurs français au XVIII et $X I X^{e}$ siècles. Parigi: Laffont.

Jonard, N. (1994). La France et l'Italie au siècle des Lumières. Essai sur les échanges intellectuels. Paris: Champion.

Lanfredini, D. (1946). Madame de Staël e i suoi amici italiani. Rivista di letteratura moderna, 1, 189-211 e 394-412.

Lanfredini, D. (1947). Madame de Staël e i suoi amici italiani. Rivista di letteratura moderna, 2, 281-297.

Manzoni, A. (2000). Carteggio. Alessandro Manzoni - Claude Fauriel, premessa di E. Raimondi, a cura di I. Botta. Milano: Centro nazionale studi manzoniani.

Monti, V. (1928). Epistolario, raccolto, ordinato e annotato da Alfonso Bertoldi (2 Voll.). Firenze: Le Monnier.

Monti, V. (2012). Primo supplemento all'epistolario, raccolto, ordinato e annotato da L. Frassineti. Milano: Cisalpino.

Necker, A.-L.-G. baronessa di Staël-Holstein (1985). Correspondance générale (Vol. V.II, Le Léman et l'Italie 19 mai 1804 - 9 novembre 1805, texte établi et présenté par B. W. Jasinski). [Paris]: Hachette.

Quondam, A. (2013). La nazione e gli italiani prima della nazione. In B. Alfonzetti e M. Formica (a cura di), L'idea di nazione nel Settecento (pp. 3-29). Roma: Edizioni di Storia e Letteratura.

Renda, F. (1996). La Rivoluzione Francese e la Sicilia. In L. Carta e G. Murgia (a cura di), Francia e Italia negli anni della Rivoluzione. Dallo sbarco francese a Quarto all'insurrezione cagliaritana del 28 aprile 1794 (pp. 128-147). Roma-Bari: Laterza.

Ricuperati, G. (2003). La cultura italiana nel secondo Settecento europeo. In G. Santato (a cura di), Letteratura italiana e cultura europea tra Illuminismo e Romanticismo (pp. 33-64). Genève: Droz.

Romagnani, G. P. (2000). La rete delle relazioni epistolari nella Rovereto del Settecento. Memorie dell'Accademia Roveretana degli Agiati, s. 2, 250, vol. 3, 47-67.

Romagnani, G. P. (2011). Epistolari e carteggi nella storiografia sul Settecento. In C. Viola (a cura di), Le carte vive. Epistolari e carteggi nel Settecento (pp. 9-24). Roma: Edizioni di storia e letteratura.

Scarabello, G. (1992). L'«Ottantanove» francese visto dalla diplomazia veneziana. In R. Zorzi (a cura di), L'eredità dell'Ottantanove e l'Italia (pp. 291-306). Firenze: Olschki. 
Servais, P. e van Ypersele, L. (2007). La lettre et l'intime. L'émergence d'une expression du for intérieur dans les correspondances privées $\left(17^{e}\right.$ - 19e siècles), avec la collaboration de F. Mirguet. Louvain-la-Neuve: Bruylant-Academia.

Venturi, F. (1973). L'Italia fuori d'Italia. In Storia d'Italia Vol. III. Dal primo Settecento all'Unità (pp. 987-1481). Torino: Einaudi.

Waquet, F. (1989). Le modèle français et l'Italie savante (1660-1750). Rome: Ecole française de Rome.

Zini, M. (1930). Il Ginguené e la letteratura italiana. Giornale Storico della Letteratura Italiana, 95, 209-242, e 96, 1-38.

\title{
«DONNEZ-MOI TOUS LES DÉTAILS QUI CONCERNENT LE PAYS QUE VOUS HABITEZ: IL EST MIEN PAR LÀ». ITALIAN-FRENCH CORRESPONDENCE BETWEEN 1789 AND 1814
}

\begin{abstract}
Summary
EpistEur is a research project of the University of Padua concerning the inventory and study of the correspondence between Italy and Europe in the $18^{\text {th }}$ century. Early data confirm the expectations of the fertility and extension of the research field and improvement of the knowledge of international relationships, diplomatic history, circulation of books, cultural exchange and the history of epistolography. EpistEur wants to contribute to redesigning the emergence of the idea of Europe in the $18^{\text {th }}$ century.

Keywords: Epistolaries, Cosmopolitanism, Italy and France, $18^{\text {th }}$ century, Correspondences, Literary Correspondence.
\end{abstract}

